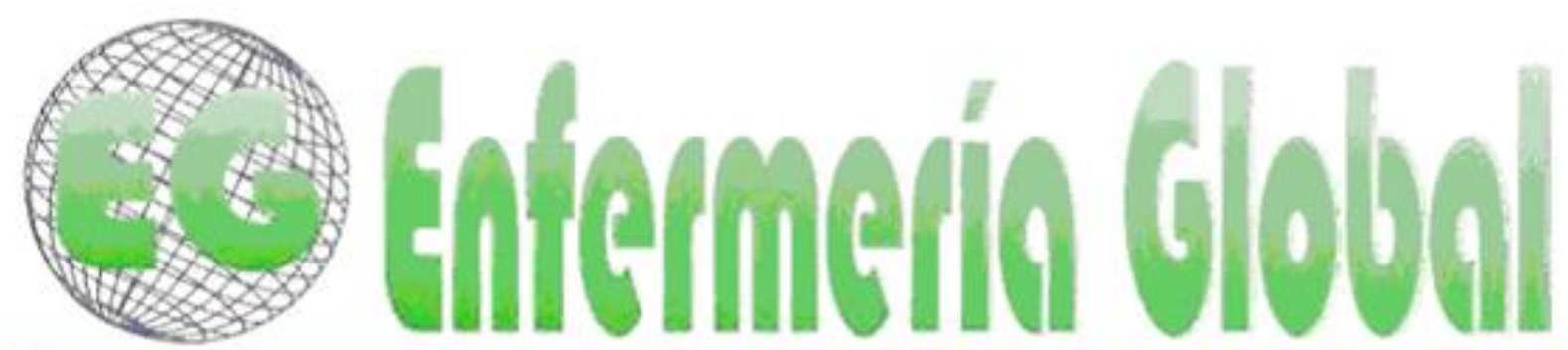

\title{
Resultados de la citología cervicovaginal en población universitaria. Un estudio descriptivo
}

Results of Papanicolaou tests in university population. A descriptive study

\section{*García Rueda, Alexandra *Fajardo Peña, María Teresa, ${ }^{* *}$ Caballero Badillo, María Claudia *Camargo-Figuera, Fabio Alberto}

\author{
*Profesor/a Escuela de Enfermería. Grupo de Investigación GRINFER. Universidad Industrial de \\ Santander. E-mail: alexagaru@hotmail.com **Jefa Sección de Servicios Integrados de Salud y \\ Desarrollo Social, Bienestar Universitario. Universidad Industrial de Santander. Colombia.
}

\author{
Palabras clave: citología; salud de la mujer; salud reproductiva \\ Keywords: Papanicolaou test; women health; reproductive health.
}

\section{RESUMEN}

Objetivo: Caracterizar los resultados de las CCV (citología cervicovaginal) de estudiantes atendidas en los servicios de Bienestar Universitario de la Universidad Industrial de Santander UIS (Bucaramanga).

Material y método: Estudio descriptivo, de corte transversal. Población: estudiantes de la UIS en el periodo comprendido entre julio de 2004 y julio de 2010. Tamaño de muestra 1543 universitarias. Para obtener la información se tuvo acceso al sistema de información de los servicios de salud de Bienestar Universitario-UIS correspondientes a los resultados de las CCV tomadas en el periodo mencionado, sistema del cual se obtuvieron las variables relacionadas con características sociodemográficas, resultados citológicos entre otras Fueron calculadas frecuencias absolutas y relativas para cada variable. Se calculó la prevalencia de anormalidad en la CCV, sus intervalos de confianza del $95 \%$. Todos los análisis fueron realizados en Stata 12.

Resultados: Conocimiento de las alteraciones en los resultados de las citologías: anormalidad: $33 \%$; principal microorganismo cándida albicans: $13.7 \%$, presencia de ASCUS:11\%, VPH: $2.5 \%$, cambios celulares reactivos:15.2\%. El $43.9 \%$ inició sus relaciones sexuales entre los 17 y 18 años, y el $23.8 \%$ inició a los 16 o menos años, el 93\% manifestó que el rango de compañeros sexuales está entre 0 y 1 y el $46 \%$ de las estudiantes utilizan métodos anticonceptivos, los más utilizados son los anovulatorios

Conclusiones: Se hallaron más factores protectores que predisponentes para presentar alteraciones citológicas y cáncer de cérvix, pero los resultados de anormalidad son significativos en la población joven, que ameritan continuar el fortalecimiento de programas enfocados a la salud sexual y reproductiva. 


\section{ABSTRACT}

Objective: Characterize the results of pap tests realized in students catered in the universitary welfare of the Universidad Industrial de Santander UIS (Bucaramanga).

Material and methods: descriptive study, of cross-section. Population: UIS students between the time frame of July 2004 and July 2010. The sample size was 1543 students. To get the information, access to the information system of universitary welfare UIS services was provided corresponding to the results of pap tests made during the time frame mencionated before. Variables related to sociodemographic characteristics, results and others were obtained too. Absolute and relative frequencies were calculated for each variable. The prevalence of abnormality was calculated and the Confidence Interval was 95\%. All the analysis was made in Stata 12.

Results: Alterations in the results of pap tests: Abnormality: 33\%; main microorganism candida albicans: $13.7 \%$, presence of ASCUS: $11 \%$, HPV: $2.5 \%$, reactive cellular changes: $15.2 \%$. $43.9 \%$ began sexual relationships between the ages of 17 and $18,23.8 \%$ said they began at the age of 16 or less, $93 \%$ manifest the range of sexual partners was between 0 and 1 and $46 \%$ of students utilize contraception methods, the most used was anovulatory.

Conclusions: More protective factors than predisposing factors to present cytological abnormalities and cervical cancer were found. But the abnormality results were significant, this deserves to continue the strengthen of programs related to sexual and reproductive health.

\section{INTRODUCCIÓN}

La citología es un examen de bajo costo, sencillo y asequible a toda la población femenina, además tiene gran especificidad en el diagnóstico de las displasias y diversos microorganismos patógenos vaginales, lo que la convierte en una gran prueba de tamizaje ${ }^{(1)}$ que ha sido utilizada tanto en el ámbito internacional como nacional en los diversos programas, estrategias de salud dirigidos a la disminución de infecciones, enfermedades de transmisión sexual y cáncer de cérvix.

Con el fin de mejorar la salud sexual y reproductiva y promover el ejercicio de los Derechos Sexuales y Reproductivos para toda la población, en el país se estableció la Política Nacional de Salud Sexual y Reproductiva en el $2003^{(2)}$ que hace especial énfasis en la reducción de los factores de vulnerabilidad y los comportamientos de riesgo, el estímulo de los factores protectores y la atención a los grupos con necesidades específicas. Esta política se propone, entre algunas metas, alcanzar coberturas de detección temprana con citología cervicovaginal (CCV) en el 90\% de la población objeto.

El estudio citológico es de gran utilidad en la detección de infecciones, reducción de muertes por cáncer a nivel cervicouterino, por lo cual es indispensable que esté acompañado de análisis de los factores de riesgo que influyen en los resultados, de programas de salud bien implementados y de políticas públicas acordes con la magnitud del problema, que permitan disminuir este tipo de infecciones y enfermedad que están afectando en todo el mundo la salud de la mujer.

Las infecciones de transmisión sexual son un problema de salud pública a nivel mundial por la "repercusión que tienen en la calidad de vida, la salud reproductiva y la salud del niño, por su función facilitadora de la transmisión sexual del VIH y su impacto en las economías nacionales e individuales" (2). Se pueden presentar desde infecciones agudas leves hasta lesiones que ocasionen secuelas desfigurativas, psicológicas, que afectan la productividad de la persona, además la atención implica una serie de costos relacionados con consultas médicas o atención de otros 
profesionales, insumos, exámenes de laboratorios, tratamientos farmacológicos, además del tiempo que la persona dura sin actividad productiva entre los trámites para recibir atención en salud y la incapacidad por la enfermedad.

Los esfuerzos de estrategias, programas y planes, realizados por parte de las instituciones prestadoras de servicios de salud están dirigidos principalmente al diagnóstico y tratamiento de este tipo de enfermedades y como las estadísticas lo demuestran las cifras siguen aumentando, por lo cual se considera que se deben implementar y fortalecer actividades hacia la prevención de los factores de riesgo para estos eventos y hacia la promoción de estilos de vida saludables de manera que se pueda intervenir en el proceso continuo de salud-enfermedad en mujeres adolescentes y jóvenes sanas.

El objetivo del presente estudio fue caracterizar los resultados de las CCV de estudiantes atendidas en los servicios de salud de Bienestar Universitario de la Universidad Industrial de Santander en Bucaramanga, Colombia.

\section{MÉTODO}

Se realizó un estudio descriptivo de corte transversal. La población de estudio estuvo conformada por las estudiantes de la Universidad Industrial de Santander. Se consideraron como criterios de inclusión: ser estudiantes que se hayan tomado la CCV en el Programa de Prevención del Cáncer de Cérvix de la Sección Servicios de Salud de la División de Bienestar Universitario de la Universidad Industrial de Santander, en el periodo comprendido entre Julio de 2004 y Julio de 2010; estudiantes en las cuales la información de los resultados de la CCV se encuentre disponible en el sistema de información de los servicios de salud de Bienestar Universitario UIS-BU UIS. Fueron excluidas del presente análisis las citologías reportadas con muestra no satisfactoria. Durante el periodo mencionado fueron seleccionadas la totalidad de las estudiantes que cumplían con los criterios anteriormente mencionados.

Para obtener la información se tuvo acceso al sistema de información de los servicios de salud de BU-UIS correspondientes a los resultados de la CCV tomadas en el periodo mencionado, sistema del cual se obtuvieron variables relacionadas con algunas características sociodemográficas, antecedentes, historia de salud sexual y reproductiva, los resultados de la CCV y algunos posibles factores relacionados a la alteración en dichos resultados. Las personas encargadas de la recolección de la información fueron los investigadores.

La variable principal fue la alteración en los resultados de la citología, desglosados en los siguientes grupos de eventos: presencia de microrganismos, anormalidades de células escamosas, otros hallazgos no neoplásicos y anormalidades en células glandulares. Otras variables utilizadas para caracterizar los resultados de las CCV fueron: edad, menarquia, estado civil, carrera que esté cursando, edad de inicio de relaciones sexuales, numero de compañeros sexuales, número de gestaciones, embarazo actual, número de partos, número de cesáreas, número de abortos, uso de métodos de planificación y recibir tratamiento hormonal.

Se realizó un análisis descriptivo de las características de la población de estudio y las características de las alteraciones de los resultados de las citologías. Fueron calculadas frecuencias absolutas y relativas para cada una de las variables 
estudiadas. Para dar cumplimiento con el objetivo del presente estudio se calculó la prevalencia de anormalidad en la CCV con sus respectivos intervalos de confianza del 95\%. Todos los análisis fueron realizados en Stata 12.

La presente investigación se concibió como una investigación sin riesgo, en el marco de la resolución 008430 de 1993 para la investigación con seres humanos, debido a que el presente es un estudio que emplea los datos de la información recolectada previamente por el sistema de información de los servicios de salud de BU-UIS manejados con confidencialidad por los investigadores, no requiriendo así la obtención de consentimiento informado por parte de las estudiantes. La información obtenida fue manejada bajo los principios éticos para investigación en salud ${ }^{(3,4,5)}$, haciendo énfasis, para este estudio, en la confidencialidad de la información, donde en la base de datos no aparecieron nombres, ni ninguna información para identificar a las participantes y a cuya base de datos solo tuvieron acceso los investigadores del estudio. El estudio contó con la revisión y aprobación por parte del comité de ética de la Facultad de Salud UIS y por las autoridades de BU-UIS correspondientes.

\section{RESULTADOS}

\section{Características sociodemográficas de la población}

Con relación a la edad de las participantes se identificó que el 30.7\% se encuentran entre 21 y 22 años y el $25.9 \%$ son menores de 20 años; respecto al estado civil el $98.4 \%$ son solteras, en su mayoría pertenecientes a la facultad de ciencias humanas con un $31 \%$, seguida por un $25.3 \%$ de la facultad de ingenierías fisicomecánicas, y $14.4 \%$ a la facultad de salud. Ver Tabla I.

Tabla I. Descripción de las características sociodemográficas de la población de estudio, $n=1543$

\begin{tabular}{lcc}
\hline \multicolumn{1}{c}{ Variable } & N & $\%$ \\
\hline Estado Civil & & \\
$\quad$ Soltera & 1519 & 98.4 \\
Otros & 24 & 1.6 \\
Facultad & & \\
Ciencias & 74 & 4.8 \\
Ciencias Humanas & 479 & 31.0 \\
Ingenierías Fisicomecánicas & 391 & 25.3 \\
Ingenierías Fisicoquímicas & 377 & 24.4 \\
Salud & 222 & 14.4 \\
Edad (años) & & \\
$\quad$ Primer Cuartil $(\leq 20)$ & 400 & 25.9 \\
Segundo Cuartil (21-22) & 474 & 30.7 \\
$\quad$ Tercer Cuartil $(23-24)$ & 366 & 23.7 \\
$\quad$ Cuarto Cuartil $(\geq 25)$ & 303 & 19.6 \\
\hline
\end{tabular}

\section{Características ginecológicas}

Como se indica en la Tabla II, el 68\% de las estudiantes tuvo su primera menstruación entre los 12 y 14 años de edad. El 43.9\% inició sus relaciones sexuales entre los 17 y 18 años y el $23.8 \%$ dio inicio a los 16 o menos años; el 93\% manifestó que el rango de compañeros sexuales está entre cero y uno. El $46 \%$ de las estudiantes utilizan 
métodos anticonceptivos, los más utilizados son los anovulatorios con un $70.7 \%$, el tiempo que llevan utilizando métodos anticonceptivos es menor o igual a seis meses (37.1\%), seguido entre 7 y 12 meses por el $23.3 \%$. No reciben tratamiento hormonal el $98.5 \%$.

\begin{tabular}{|c|c|c|}
\hline Variable & $\mathbf{n}$ & $\%$ \\
\hline \multicolumn{3}{|c|}{ Uso actual de anticonceptivo } \\
\hline No & 832 & 53.9 \\
\hline $\mathrm{Si}$ & 711 & 46.1 \\
\hline \multicolumn{3}{|l|}{ Tipo de Anticonceptivo } \\
\hline Anovulatorio & 503 & 70.7 \\
\hline Otro & 175 & 24.6 \\
\hline DIU & 30 & 4.2 \\
\hline Quirúrgico & 4 & 0.6 \\
\hline \multicolumn{3}{|c|}{ Tiempo de Uso de Anticonceptivo } \\
\hline 6 meses o menor & 264 & 37.1 \\
\hline Entre 7 y 12 meses & 166 & 23.3 \\
\hline Entre 13 y 24 meses & 156 & 21.9 \\
\hline 25 meses o mayor & 126 & 17.7 \\
\hline \multicolumn{3}{|c|}{ Recibe Tratamiento Hormonal } \\
\hline $\begin{array}{l}\text { No } \\
\mathrm{Si}\end{array}$ & $\begin{array}{c}1520 \\
23\end{array}$ & $\begin{array}{c}98.5 \\
1.5\end{array}$ \\
\hline \multicolumn{3}{|l|}{ Edad de la Menarca } \\
\hline 11 años o menor & 370 & 24.1 \\
\hline Entre 12 y 14 años & 1044 & 68.0 \\
\hline 15 años o mayor & 121 & 7.9 \\
\hline \multicolumn{3}{|c|}{ Edad de Inicio de Relaciones Sexuales } \\
\hline 16 años o menor & 351 & 23.8 \\
\hline Entre 17 y 18 años & 649 & 43.9 \\
\hline Entre 19 y 20 años & 342 & 23.1 \\
\hline 21 años o mayor & 136 & 9.2 \\
\hline \multicolumn{3}{|c|}{ Numero de compañeros sexuales } \\
\hline Ninguno o 1 & 854 & 93.0 \\
\hline 2 o más & 64 & 7.0 \\
\hline
\end{tabular}

\section{Características obstétricas}

Sobre embarazo actual, la Tabla III señala que el $98.6 \%$ de las estudiantes no están en gestación. Igualmente no tienen antecedentes de: gestación el $92.8 \%$, partos el 97.3, ni de cesáreas el $98.5 \%$. 


\begin{tabular}{|c|c|c|}
\hline Variable & $\mathbf{n}$ & $\%$ \\
\hline \multicolumn{3}{|c|}{ Embarazo Actual o Tiene Duda } \\
\hline No & 1521 & 98.6 \\
\hline $\mathrm{Si}$ & 22 & 1.4 \\
\hline \multicolumn{3}{|c|}{ Antecedentes: embarazos } \\
\hline Ninguno & 1431 & 92.8 \\
\hline 1 o más & 111 & 7.2 \\
\hline \multicolumn{3}{|l|}{ Antecedentes: partos } \\
\hline Ninguno & 1501 & 97.3 \\
\hline 1 o más & 41 & 2.7 \\
\hline \multicolumn{3}{|l|}{ Antecedentes: abortos } \\
\hline Ninguno & 1487 & 96.4 \\
\hline 1 o más & 55 & 3.6 \\
\hline \multicolumn{3}{|l|}{ Antecedentes: cesáreas } \\
\hline Ninguno & 1519 & 98.5 \\
\hline 1 o más & 23 & 1.5 \\
\hline
\end{tabular}

\section{Resultados de las citologías}

De los resultados de las citologías realizadas a las estudiantes del estudio, la Tabla IV presenta los siguientes hallazgos, se encontró que el 33\% fueron anormales, clasificación compuesta por los resultados de: anormalidades en células escamosas, anormalidades en células glandulares y otros hallazgos no neoplásicos, dentro de esta clasificación el mayor porcentaje fue del $18 \%$ por la presencia de anormalidades de células escamosas es decir agrupadas por: VPH(2.5\%), ASC-US(11\%) ASC$H(0.3 \%)$, Lesión intraepitelial escamosa de bajo grado (LIE BG) $(6.4 \%)$, lesión intraepitelial escamosa de alto grado (LIE AG) $(0.4 \%)$, lesión intraepitelial escamosa de alto grado sospechosa de infiltración (0\%) y carcinoma escamolecular $(0 \%)$, seguida por el $15.4 \%$ con la presencia de otros hallazgos no neoplásicos, en los cuales se identificó cambios celulares reactivos, cambios celulares reactivos asociados a radiación, cambios celulares asociados a DIU, atrofia, células endometriales, células glandulares poshisterectomía y solo el $0.1 \%$ presentó anormalidades en células glandulares.

Igualmente cabe resaltar que el $13.9 \%$ de los resultados de citologías anormales fue dado por la presencia de microorganismos, dentro de los cuales, la candida albicans representa el $13.7 \%$. 


\section{Tabla IV. Prevalencias y sus intervalos de confianza del $95 \%$ de los resultados de la citología de la población de estudio, $n=1543$}

\begin{tabular}{lccc}
\hline \multicolumn{1}{c}{ Variable } & $\mathbf{n}$ & $\%$ & IC $95 \%$ \\
\hline Resultado anormal & 517 & 33.5 & $31.2 ; 35.9$ \\
Presencia de microorganismos & 214 & 13.9 & $12.2 ; 15.7$ \\
Trichomonas & 1 & 0.1 & $0.002 ; 0.4$ \\
Cándida & 211 & 13.7 & $12.0 ; 15.5$ \\
Actínomices & 0 & 0 & $0 ; 0.2$ \\
Herpes & 4 & 0.3 & $0.07 ; 0.7$ \\
Presencia de anormalidades de células escamosas & 279 & 18.1 & $16.2 ; 20.1$ \\
Positivo para VPH & 39 & 2.5 & $1.8 ; 3.4$ \\
ASC-US & 169 & 11.0 & $9.4 ; 12.6$ \\
ASC-H & 4 & 0.3 & $0.07 ; 0.7$ \\
LIE BG & 98 & 6.4 & $5.2 ; 7.7$ \\
LIE AG & 6 & 0.4 & $0.1 ; 0.8$ \\
LIE AG sospechosa de infiltración & 0 & 0 & $0 ; 0.2$ \\
Carcinoma escamolecular & 0 & 0 & $0 ; 0.2$ \\
Presencia de otros hallazgos no neoplásicos & 238 & 15.4 & $13.7 ; 17.3$ \\
Cambios celulares reactivos & 234 & 15.2 & $13.4 ; 17.1$ \\
Cambios celulares reactivos asociados a radiación & 1 & 0.1 & $0.002 ; 0.4$ \\
Cambios celulares asociados a DIU & 3 & 0.2 & $0.04 ; 0.6$ \\
Atrofia & 0 & 0 & $0 ; 0.2$ \\
Células endometriales & 0 & 0 & $0 ; 0.2$ \\
Células glandulares poshisterectomía & 0 & 0 & $0 ; 0.2$ \\
Anormalidades en células glandulares & 2 & 0.1 & $0.02 ; 0.5$ \\
Células endocervical atípicas & 2 & 0.1 & $0.02 ; 0.5$ \\
Células endometriales atípicas & 0 & 0 & $0 ; 0.2$ \\
Células glandulares atípicas & 0 & 0 & $0 ; 0.2$ \\
Células endocervical atípicas sospechosas de malignidad & 0 & 0 & $0 ; 0.2$ \\
Células endometriales atípicas sospechosas de malignidad & 0 & 0 & $0 ; 0.2$ \\
Células glandulares atípicas sospechosas de malignidad & 0 & 0 & $0 ; 0.2$ \\
Adenocarcinoma endocervical IN SITU & 0 & 0 & $0 ; 0.2$ \\
Adenocarcinoma endocervical & 0 & 0 & $0 ; 0.2$ \\
Adenocarcinoma endometrial & 0 & 0 & $0 ; 0.2$ \\
Adenocarcinoma extrauterino & 0 & 0 & $0 ; 0.2$ \\
Adenocarcinoma no especificado & 0 & 0 & $0 ; 0.2$ \\
\hline
\end{tabular}

\section{DISCUSIÓN}

Al comparar y analizar los hallazgos de la investigación con otros estudios se identifican aspectos importantes descritos a continuación que se correlacionan con los resultados de las citologías:

El alto porcentaje de mujeres del estudio con ninguno o un compañero sexual estable, es un factor protector para no desarrollar cáncer cervicouterino, porque según estudios ${ }^{(6,7)}$ el hecho de tener seis o más compañeros sexuales es un factor de riesgo para la génesis del cáncer de cérvix. Igualmente este factor protector puede estar relacionado con el bajo porcentaje de VPH, pues el factor de riesgo más consistente y documentado para la infección por VPH es el número de compañeros sexuales ${ }^{(8,9)}$

La edad de inicio de las relaciones sexuales entre los 17 y 18 años de edad, por parte de las estudiantes del estudio, es similar a otro estudio efectuado en Bogotá ${ }^{(10)}$, en el cual iniciaron estas relaciones entre los 15 y 18 años 67.5\%. Estos resultados se soporta en los datos estadísticos de la Encuesta Nacional de Demografía y Salud del $2010^{(11)}$ en la cual esta encuesta reporta una edad mediana similar a la primera relación sexual para las mujeres de 20 a 49 años correspondiente a 18 años y el 
$13,5 \%$ de las mujeres entre 15 y 19 años tuvieron su primera relación antes de cumplir los 15 años de edad, el $48 \%$ de las mujeres entre 25 y 49 años tuvieron su primera relación sexual antes de los 18 años y el 70 por ciento antes de cumplir los 20 años. Es decir, que la primera relación sexual en las mujeres colombianas se está dando a más temprana edad.

Igualmente, con el inicio de las relaciones sexuales, las mujeres están propensas a adquirir enfermedades de transmisión sexual, de las cuales el VPH es una de las más comunes, según un estudio realizado en Venezuela ${ }^{(12)}$ se encontró que el $50 \%$ de las jóvenes con edad comprendida entre 17-19 años tenían infección por VPH y las pacientes con diagnóstico de NIC I presentaron VPH en el 80,9\% de los casos; por su parte, Winer Rachel y colaboradores ${ }^{(13)}$ encontraron en su investigación que la incidencia de infección genital por VPH fue de 37.8 por cada 100 pacientes-año. Sin embargo, aunque estos resultados difieren de los encontrados en el presente estudio, pues solo el $2.5 \%$ de las estudiantes presentó $\mathrm{VPH}$, no hay que desconocer sus alcances, porque es más frecuente en mujeres jóvenes sexualmente activas $(14,15)$ su papel oncogénico se ha establecido sobre todo en relación con el cáncer del cuello uterino $^{(16)}$.

Estudios consideran que solo la infección por el VPH no puede ser suficiente para promover la carcinogénesis cervical, porque hay otros factores que podrían estar relacionados, como la deficiencia de vitamina $A$, el tabaquismo, anticonceptivos orales y otras enfermedades de transmisión sexual ${ }^{(17)}$, como el Virus del Herpes Simple (HSV), las infecciones por Chlamydia trachomatis, la inmunosupresión prolongada ${ }^{(18,19)}$ bajo nivel educativo y socioeconómico ${ }^{(20)}$; de los cuales para la población de nuestro estudio, vienen siendo factores protectores el tiempo de uso de anticonceptivos orales menor de 6 meses y el nivel educativo y socioeconómico por ser estudiantes universitarias y el bajo porcentaje de herpes. En las variables del estudio, una limitante fue que no se tuvo en cuenta la deficiencia de vitamina $A$, inmunosupresión y tabaquismo.

El $92.8 \%$ de las estudiantes no tenía antecedentes de embarazo, dato favorable para las mujeres del estudio, teniendo en cuenta que la mayoría son adolescentes, jóvenes y un embarazo a temprana edad puede ocasionar grandes riesgos, dificultades a nivel psicológico, social, profesional y en especial para el caso, riesgos biológicos, porque sus organismos aún no están acondicionados vivos es un factor de riesgo para cáncer de cuello uterino ${ }^{(21)}$

El porcentaje de citologías con resultado anormal fue mayor (33.5\%), en comparación con otros estudios en el ámbito nacional e internacional, realizados en grupos de estudiantes universitarias en los cuales la prevalencia de alteraciones citológicas fue del $10 \%$ en Popayán ${ }^{(22)}$, y en mujeres jóvenes y adolescentes en Estados Unidos y Canadá reportar $11.7 \%$ y $13 \%$ respectivamente ${ }^{(23,24)}$, aunque al comparar estos estudios se identifica que el tamaño de las muestras es inferior al presentado en este estudio (1543), que pueden influir en el menor porcentaje de prevalencia de anormalidad citológica, por ejemplo en Popayán la muestra fue de 67 estudiantes universitarias y en Canadá la muestra fue de 871 adolescentes; es claro en el análisis de los estudios el incremento de alteraciones en las citologías, por tal razón es necesario intervenir los factores de riesgo que están relacionados con estas alteraciones. 
Las alteraciones del estudio más significativas que contribuyeron al porcentaje de anormalidad citológica fueron la presencia de microorganismos(13.9\%) y de células escamosas atípicas de significado indeterminado ASCUS (11\%), que al contrastar con otros estudios fue mayor el porcentaje en éstos, como el de Alteiro y colaboradores (25), quienes identificaron ASCUS en el $22 \%$ de las citologías, asimismo, González y colaboradores $^{(26)}$ hallaron ASCUS en el $24.7 \%$; Massad y colaboradores ${ }^{(27)}$ el $27 \%$, sin embargo en otros estudios ocurrió lo contrario, los autores hallaron porcentajes menores de ASCUS, tal fue el caso de Bravo $5 \%{ }^{(22)}$, Mount $9.7 \%{ }^{(23)}$ y Mangan $4.1 \%$ (24). Estudios han observado que concomitante con el ASCUS puede hallarse una lesión de alto grado subyacente, cuya prevalencia, según el tipo de estudio, puede variar entre 5 y $63 \%{ }^{(28,29)}$.

Llama la atención que el microorganismo másfrecuente en los resultados de las citologías fue la Cándida con $13.7 \%$, pues al revisar la literatura son pocas las investigaciones de este tipo que refieren hallazgos de este hongo (levadura) ${ }^{(25)}$, el cual hace parte de la flora normal, pero que ocasiona infección cuando hay crecimiento desmedido de Cándida; que se produce al haber un desequilibrio mínimo en los cambios de acidez normal de la vagina o cambios en el equilibrio hormonal. ${ }^{(30)}$. Tales factores son suficientes para que las levaduras se multipliquen ${ }^{(31,32,33)}{ }^{\text {. Es por }}$ ello importante fortalecer en esta población medidas preventivas para la candidiasis, especialmente el uso de preservativo para evitar el contagio sexual, así como el tratamiento farmacológico. Igualmente se ha encontrado que las infecciones por otros microorganismos son más comunes, según la OMS y colaboradores ${ }^{(34)}$ cada año se registran en todo el mundo más de 340 millones de casos nuevos de infecciones bacterianas y protozoarias de transmisión sexual.

Finalmente, para próximos estudios, se sugiere tener en cuenta variables como el hábito de fumar, el uso de preservativos, los cuales fueron una limitante del estudio, pues no estaban incluidos en el formato de citologías.

\section{CONCLUSIONES}

De acuerdo a los resultados obtenidos, se identifica que en la mayoría de las estudiantes del estudio se encuentran más factores protectores que predisponentes para presentar alteraciones citológicas y cáncer de cérvix.

Asimismo, la anormalidad en los resultados de la citología ocurrió principalmente por microorganismos y presencia de anormalidades de células escamosas, los cuales son datos significativos en la población joven que ameritan la necesidad de continuar el fortalecimiento de los programas enfocados a la promoción de la salud sexual y reproductiva, prevención, detección y tratamiento de enfermedades cervico uterinas.

\section{REFERENCIAS}

1. Rivera B, Quintero J. Prevalencia de lesiones intraepiteliales escamosas y factores de riesgo en las usuarias del servicio de citologías del laboratorio docente asistencial, proyecto de investigación, Escuela de Bacteriología, Universidad de Antioquia, Medellín 2006.

2. Ministerio de Protección Social, Fondo de Población de las Naciones Unidades UNFPA, Instituto PROINAPSA UIS. Servicios de salud amigables para jóvenes y adolescentes. Colombia 2007p.4-95. 
3. Ministerio de Salud. Resolución 8430. Normas científicas, técnicas y administrativas de investigación en salud. Colombia. 1993.

4. Asociación Médica Mundial. Declaración de Helsinki. Principios éticos para las investigaciones médicas en seres humanos. Escocia, Octubre 2000.

5. Council for international organizations of medical sciences. Pautas éticas internacionales para la investigación biomédica en seres humanos. Geneva: Council for international organizations of medical sciences; 2002

6. Institute for clinical sistems improvement. health care guideline: cervical cáncer screening: Citado por: Amaya J, Restrepo S, Tamizaje para cáncer de cuello uterino: cómo, desde y hasta cuándo. en: rev. colombiana de ginecología y obstetricia. 2005, vol.56 (1):59-67

7. Grisales H, Vanegas A, Gaviria A, Castaño J, Alonso M, Borrero M; et al. Prevalencia de anormalidades de células epiteliales y factores asociados en mujeres de un municipio rural Colombiano. Instituto Nacional de Colombia. Revista biomédica Biomédica 2008; 28:271-83

8. Muñoz N, Méndez F, Posso H, Molano M, Van den Brule AJ, Ronderos M, Meijer C, Muñoz A. Incidence, duration, and determinants of cervical human papillomavirus infection in a cohort of Colombia women with normal cytological results. J Infect Dis. 2004; 190:2077-87.

9. Tafurt Y, Acosta C, Sierra C, Prevalencia de citología anormal e inflamación y su asociación con factores de riesgo para neoplasias del cuello uterino en el Cauca, Universidad del Cauca Popayán, Colombia. Rev. salud pública. 2012,14 (1): 53-66 10. Acosta $\mathrm{S}$. et.al Conductas de salud y factores de riesgo en la salud sexual y reproductiva de una población universitaria. Rev. Ciencias biomédicas. Enero a junio de 2010; 8(13):32-43 Disponible en línea: http://www.unicolmayor.edu.co/invest_nova/.../NOVA13_ARTORIG3

11. Profamilia. Encuesta nacional de demografía y salud- ends. Bogotá: Profamilia. 2010 :148.disponibleenlinea :

http:/WWW.PROFAMILIA.ORG.CO/ENCUESTAS/PROFAMILIA/PROFAMILIA/INDE X.PHP?.

12. Alfonzo B, et al. Detección del virus papiloma humano en muestras cervicales de una población de estudiantes de la universidad central de Venezuela, Revista de la facultad de medicina RFM, Caracas 2003. Vol.26 (2):5-10.

13. Winer R. et al. El uso de condón y el riesgo de infección genital del virus de papiloma humano en mujeres jóvenes. en: The new england journal of medicine. junio, Washigton. 2006; 354(25) P.2648.

14. Seltman, Castle P, Guido R, Schiffman M, Wheeler C, et al. Smoking is a risk factor for cervical intraepithelial neoplasia grade 3 among oncogenic human papillomavirus dna-positive women with equivocal or mildly abnormal cytology. en: Cáncer epidemiolbiomarkersprev. 2005, (14):2.

15. Romero Ivette, Ceballos Clarena, Monterrora Álvaro. Lesiones premalignas y malignas de cérvix en adolescentes y mujeres jóvenes. en: rev. Medunab. Cartagena. Colombia 2009. 12 (1):17.

16. Ybarra L, Pérez B, Romero D. Conocimiento y creencias sobre las prueba de papanicolau en estudiantes universitarios. Rev. Psicología y Salud, julio-diciembre de 2012 (22)2:185-194, Disponible en: http://www.uv.mx/psicysalud/psicysalud-22-2/222/José\%20Luis\%20Ybarra\%

17. Lukic A, Canzio C, Patella A, Giovagnoli M, Cipriani P, Frega A, Moscarini M. "Determination of Cervicovaginal Microorganisms in Women with Abnormal Cervical Cytology: The Role Of Ureaplasmaurealyticum". En: Anticancer Research. 2006. Vol. 26. p4843. 
18. Abdullgaffar $\mathrm{B}$, Alsaleh $\mathrm{J}$, Hattawi $\mathrm{H}$. The prevalence of abnormal cervical cytology in women with systemic lupus erithematosus.diagn. Cytopathol. $2012 \mathrm{apr} ; 40(4): 327-31$ 19. American college of obstetricians and gynecologists. practice bulletin number 109. cervical cytology screening. obstetgynecol 2009;114:1409-20.

20. Khan M, Patridge E, Wang S, Schiffman M. Socioeconomic status and the risk of cervical intraepithelial neoplasia grade 3 among oncogenic human papillomavirus dnapositive women with equivocal or mildly abnormal cytology. The american cáncer society. Cáncer 2005; 104(1):61-70

21. Piñeros $M$, et al, cobeCrtura de la citología de cuello uterino y factores relacionados en Colombia. 2005. Instituto nacional de cancerología. Bogotá en: Revista de salud pública. 2007. 9 (3):327-341

22. Bravo $\mathrm{M}$ et al. Prevalencia de anormalidades en la citología cervical en tres grupos poblacionales de Popayán Colombia 2003-2005, en: rev. colombiana de obstetricia y ginecología. 2008. 59(3):190-198.

23. Mount SL, Papillo JL. A study of 10,296 pediatric and adolescent papanicolaou smear diagnoses in northern new england. Pediatrics 1999;103:539-545.

24. Mangan S, Legano L, Rosen C, McHugh M, Fierman A, Dreyer B, et al. Increased prevalence of abnormal Papanicolaou smears in urban adolescents. Arch Pediatr Adolesc Med 1997;151:481-484.

25. Alteiro $G$, et al. Hallazgos citológicos y factores de riesgo para patología preinvasora e invasora de cuello uterino. Área de influencia del ambulatorio urbano tipo II "Dr. Rafael Pereira". Barquisimeto, Estado Lara, Rev. Salud pública y nutrición. Venezuela. 2007; 8 (3):1-8

26. González M., Murillo R., Osorio E. Gamboa O. Ardila J. Pprevalencia de anormalidades citológicas e histológicas de cuello uterino en un grupo de mujeres en Bogotá. rev colomb cancerol.Colombia. 2010;14(1):22-28

27. Massad S, Markwell S, Cejtin H, Collins Y. Risk of high grade cervical intraepitelial neoplasia among young women with abnormal screening cytology. American society for colposcopy and cervical pathology journal of lower genital tract disease, 2005;9:(4):225-229. disponible en línea: http://www.ncbi.nlm.nih.gov/pubmed?linkname..uid

28. Kulasingam S, Hughes J, Kiviat N, Mao C, Weiss N, Kuypers J, et al. Evaluation of human papillomavirus testing in primary screening for cervical abnormalities: comparison of sensitivity, specificity, and frequency of referral. JAMA 2002;288:17491757.

29. Apgar B, Soschnick L, Wright TC Jr. The 2001Bethesda System Terminology. Am Fam Physician 2003; 68:1992-1998.

30. Buitrón R., Bonifaz A., Amancio O., Basurto E., Araiza J., Romero R. Correlación clínico-micológica de la candidiasis vulvovaginal, Ginecol Obstet Mex México. 2007;75:68-72

31. Bonifaz A. Candidiasis. En: Micología Médica Básica. 2aㅡㄹ Edición. México: Méndez Editores, 2000:301-330.

32. Fah S, Wong B. Current status of nonculture methods for diagnosis of invasive fungal infections. Clin Microbiol Rev. 2002;15:465-484.

33. Moragues MD, Omaetxebarria MJ, Elguezabal N, Bikandi J, Quindós G. Serological differentiation of experimentally induced Candida dubliniensis and Candida albicans infections. J Clin Microbiol Rev.2001;39:2999-3001.

34. Organización Mundial de la Salud, et al. Estrategia mundial de prevención y control de las infecciones de transmisión sexual, 2006-2015: Romper la cadena de transmisión. $2007 . \quad$ p.1-11 Disponible en línea: http://www.bvs.sld.cu/revistas/gin/vol34_1_08/gin07108.htm 
Recibido: 5 de noviembre 2014; Aceptado: 24 de enero 2015 